LA GRANJA:

REVISTA DE

CIENCIAS DE LA VIDA

pISSN:1390-3799; eISSN:1390-8596

http:/ / doi.org/10.17163/lgr.n26.2017.04
Artículo científico / Scientific paper

CONSERVACIÓN

\title{
CARACTERIZACIÓN MORFOLÓGICA, CULTURAL Y PATOGÉNICA DE AISLADOS DE colletotrichum sp. PRODUCIENDO ANTRACNOSIS EN MANGO (mangifera indica L.).
}

\author{
CHARACTERIZATION MORPHOLOGICAL, CULTURAL AND PATHOGENIC OF \\ ISOLATED colletotrichum sp. ANTHRACNOSE PRODUCING IN MANGO
}

(mangifera indica L.).
Luis Alberto Valdés ${ }^{1}$; Diana Calero Consuegra ${ }^{1}$, Adys Gómez ${ }^{2}$, María Elena Carballo $^{3}$, Maricela Capote ${ }^{2}$, Ingrid González ${ }^{6}$, José Miguel Alvarez ${ }^{5}$, Rohde $\mathrm{W}^{4}$

${ }^{1}$ Universidad Politécnica Salesiana. Quito. Ecuador. Av. Isabel La Católica N. 23-52 y Madrid. Quito, Ecuador.

2 Instituto de Investigaciones en Fruticultura Tropical (IIFT). Calle 7 ma número 5005 entre 30 y 32. Playa. La Habana, Cuba.

${ }^{3}$ Facultad de Biología, Universidad de La Habana. Calle 25 No. 455 Vedado. La Habana, Cuba.

${ }^{4}$ Max Planck Institute for Plant Breeding Research (MPI). Carl-von-Linne-Weg 10, 50829 Köln, Alemania.

${ }^{5}$ Universidad de Las Américas (UDLA). Av. de los Granados E12-41 y Colimes. Quito, Ecuador.

${ }^{6}$ Instituto de Biotecnología. UNAM Avenida Universidad 2001, Chamilpa, 62210 Cuernavaca, Morelos, México.

*Autor para correspondencia: dcalero@ups.edu.ec

Artículo enviado el 17 de abril de 2017. Aceptado, tras revisión, el 28 de abril de 2017. Publicado el 1 de septiembre de 2017.

\begin{abstract}
Resumen
La antracnosis es considerada la principal enfermedad fúngica que afecta al mango. Causa considerables daños en las hojas, las flores y los frutos. Los hongos filamentosos del género Colletotrichum y su teleomorfo Glomerella son considerados los patógenos de plantas más ampliamente distribuidos a nivel mundial, causantes de esta enfermedad. En esta investigación se obtuvieron 22 aislados del género Colletotrichum de diferentes localidades de la región occidental país, en seis variedades de mango. La caracterización cultural fue diversa en el crecimiento de colonias en PDA, con presencia y ausencia de exudaciones a diferentes temperaturas, siendo los 24 grados la óptima para el crecimiento. La fase sexual observada en algunos aislados muestra pocos peritecios. La concentración de conidios varió entre 107 y 105 medidos Cámara de Neubauer mientras los apresorios y conidios están dentro de los rangos para la especie Colletotrichum gloeosporiodes. La variabilidad en la patogenicidad mostró que todas las especies de mangos investigadas son susceptibles al microorganismo. El análisis estadístico arroja el agrupamiento de los aislados por localidad con características patogénicas similares.
\end{abstract}

Palabras claves: Glomerella cingulata, antracnosis, apresorios, conidios, patogenicidad. 


\begin{abstract}
Anthracnose is considered the main fungal disease affects mango. Cause considerable damage to leave, flowers and fruits. The filamentous fungi of the genus Colletotrichum and its teleomorph Glomerella pathogens are considered the most widely distributed plants worldwide, causing this disease. In this investigation were obtained 22 isolates of $\mathrm{Co}^{-}$ lletotrichum from different localities of the country western region, in six varieties of mango. Cultural characterization was varied in the growth of colonies on PDA, with presence and absence of exudation at different temperatures, 24 degrees being optimal for growth. The observed in isolated sexual stage shows few perithecia. Conidia concentration ranged between 107 and 105 measured Neubauer chamber while appresoria and conidia are within the ranges for the species Colletotrichum gloeosporioides. Variability in pathogenicity showed that all species investigated wizards are susceptible to microorganism. The statistical analysis shows the grouping of isolates by locality with similar pathogenic characteristics.

Keywords: Glomerella cingulata, anthracnose, appressoria, conidia pathogenicity.

Forma sugerida de citar: Valdéz, L., Calero, D., Gómez, A., Carballo, M., Capote, M., González, I., Alvarez, J., Rohde, W. 2017. Caracterización morfológica, cultural y patogénica de aislados de colletotrichum sp. produciendo antracnosis en mango (mangifera indica l.). La Granja: Revista de Ciencias de la Vida. Vol. 26(2):38-51. pISSN:1390-3799; eISSN:1390-8596.
\end{abstract}




\section{Introducción}

El mango (Mangifera indica L.) es un cultivo bien adaptado a las condiciones de Cuba (Capote et al. 1989; Valdés et al., 2015). La antracnosis es la principal enfermedad que ataca este cultivo en el país. La misma es causada por el hongo Colletotrichum gloeosporioides (Penz) Penz \& Sacc. fase perfecta Glomerella cingulata, que provoca la caída de la inflorescencia, afecta los frutos, hojas y ramas jóvenes (álvarez et al., 2006). También aparece como una enfermedad poscosecha de las frutas maduras durante el almacenamiento. Como consecuencia se obtienen malas cosechas con frutos de baja calidad (Rebouca, 2002; Bruwer et al., 2006), lo que conduce a una disminución del precio de la fruta, tanto en el mercado interno como en el internacional (Arauz, 2000; Rodríguez et al., 2002).

El control de las enfermedades producidas por el hongo representa un reto para el agricultor, donde la estrategia de protección preventiva y el factor tiempo son elementos indispensables dado el corto período de incubación del hongo y su alta capacidad de esporulación en tejidos jóvenes (Infoagro, 2006; Abd-Alla y Wafaa, 2010). Por tanto, se hace necesario integrar medidas de manejo como el uso de variedades con cierto grado de tolerancia o resistencia (Carrillo et al., 2005).

En ocasiones implementar medidas de control resulta difícil por la convergencia en un mismo hospedante de varias especies o subespecies de hongos del género Colletotrichum y la gran variedad de formas morfológicas de estas en relación con las variaciones ambientales (Santiago et al., 2005; Butt y Copping, 2000). Para intentar resolver esta situación los estudios se han focalizado, primero hacia la identificación taxonómica de especies y en segundo lugar a la caracterización de sub-poblaciones de cada especie; resultando imprescindible para la implementación de estrategias de control efectivas (Santiago et al., 2005; Jayasinghe y Fernando, 2009).

El presente trabajo tuvo como objetivo caracterizar morfológica, cultural y patogénicamente las cepas de Colletotrichum sp. que conviven en plantaciones de mango en varias de las provincias occidentales de Cuba.

\section{Materiales y métodos}

Obtención de los aislamientos: Se colectaron muestras vegetales con síntomas de antracnosis procedentes del municipio de Alquízar, provincia Habana y de parcelas particulares de los municipios Escambray y Jaruco, ubicados en las provincias Cienfuegos y La Habana respectivamente. Las porciones afectadas se lavaron con agua corriente, se desinfectaron con hipoclorito de sodio al $1 \%$ durante 1 min y se sembraron en cajas Petri con medio de cultivo Agar-Papa-Dextrosa (PDA), se incubaron a 30oC hasta que se observó crecimiento micelial. A partir de estas se realizaron cultivos monospóricos mantenidos a $30 \mathrm{oC}$ en oscuridad. Una vez crecidos, se conservaron en el cepario en tubos de ensayo a $4^{\circ} \mathrm{C}$.

Caracterización cultural de los aislamientos: Los aislamientos del cepario se sembraron en cajas Petri con PDA y se incubaron a $27 \pm 2^{\circ}$, durante 7 días. Una vez crecidas las colonias se tomaron discos con sacabocados de $5 \mathrm{~mm}$ de diámetro y se colocaron en el centro de cajas Petri con PDA, de 11 $\mathrm{cm}$ de diámetro. Se evaluaron 3 réplicas por aislado a las temperaturas 20, 24, 27 y $30^{\circ}$, durante 7 días. Pasado este tiempo se realizó la observación de las características culturales y medición de las colonias siguiendo la metodología de Freeman et al. (1998). A los datos del diámetro de la colonia se les realizó un análisis de varianza bifactorial con dos tratamientos y tres réplicas por tratamientos. Las medias fueron comparadas por el Test de Rangos Múltiples de Duncan (Cigarroa, 1985).

Observación de la fase sexual: Los aislados del cepario se sembraron en cajas de $11 \mathrm{~cm}$ de diámetro con PDA y se incubaron a $30^{\circ}$ bajo un ciclo de 12 horas luz y 12 horas oscuridad. A partir de estas cajas se realizaron observaciones semanales, por espacio de 2 meses, para determinar la formación de peritecios y ascosporas propios de la fase sexual (Glomerella cingulata).

Determinación de la concentración conidial: Para evaluar la concentración de conidios por aislado, se prepararon suspensiones adicionando 5 discos de micelio, obtenidos a partir de colonias de 15 días de incubación a $30^{\circ}$, en $10 \mathrm{ml}$ de agua destilada estéril como se describió anteriormente. A partir de estas se determinó la concentración en Cámara de Neubauer. A los datos obtenidos se les realizó un análisis de varianza de clasificación simple (ANOVA) y las medias fueron comparadas por 
el Test de rangos múltiples de Duncan (Cigarroa, 1985).

\subsection{Caracterización morfométrica de los aislamientos}

Morfometría de los conidios: A partir de colonias con 7 días de incubación a $30^{\circ}$, se tomaron 5 discos de $5 \mathrm{~mm}$ de diámetro por aislado y se adicionaron en $10 \mathrm{ml}$ de agua destilada estéril, colocándose en un vortex durante $1 \mathrm{~min}$ para propiciar la liberación de los conidios. La concentración de las suspensiones se ajustó a $1 \times 10^{5}$ conidios. $\mathrm{ml}^{-1}$. A partir de estas se hicieron preparaciones y se midieron 50 conidios por aislado, describiéndose su forma según (Gutiérrez et al., 2001). Esto se realizó con la ayuda del microscopio óptico Olympus con un aumento de $100 x$.

Morfometría de los apresorios: Se prepararon portaobjetos recubiertos con una película fina de Agar-Agua y se adicionaron $50 \mu \mathrm{l}$ de suspensiones ajustadas a $1 \times 105$ conidios. $\mathrm{ml}^{-1}$. Se realizaron tres réplicas por aislado. Los portaobjetos se incubaron a temperatura ambiente durante 24 horas, pasado este período se observaron al microscopio óptico Olympus, con aumentos de 40 y 100x, midiéndose y describiéndose 50 apresorios por cada aislado, según Gutiérrez et al., 2001.

Prueba de patogenicidad: La capacidad infecciosa de los aislamientos se evaluó en hojas de plantas de mango de las variedades Haden, Filipino, Señora y Keitt, procedentes de la colección de mango de la Estación Experimental de Frutales del municipio de Alquízar, provincia Artemisa. Se colectaron 90 hojas jóvenes y sanas de cada variedad. Estas se lavaron con agua, detergente y alcohol al $70 \%$ para eliminar suciedades y desinfectar respectivamente. Luego se colocaron en cajas Petri conteniendo portaobjetos y papel de filtro humedecido con agua estéril.

Se montaron tres réplicas y un testigo por cada variedad y cada aislado, realizando 6 incisiones con una aguja en cada hoja. Como inóculo se usó $10 \mu \mathrm{l}$ de una dilución de 1 × 105 conidios.ml $^{-1}$ para cada aislado. En el caso de los testigos, se aplicaron 10 $\mu l$ de agua destilada estéril en lugar de la suspensión. Las cajas se incubaron a temperatura ambiente y se observaron diariamente por espacio de 5 días, siguiendo la aparición de síntomas y midiendo el diámetro de la lesión (virulencia de la cepa). Los datos del diámetro de la lesión por aislado y variedad se procesaron usando la transformación $(x+1)$ y se analizaron siguiendo un análisis de varianza trifactorial. Las medias fueron comparadas por el Test de rangos múltiples de Duncan (Cigarroa, 1985).

Análisis estadístico para la agrupación de los aislados: Se realizó un análisis estadístico de componentes principales (ACP) con los resultados obtenidos para determinar la variable que más aportó a la diversidad. Se hizo un análisis de agrupamiento con las tres variables que más aportan utilizando la distancia euclidiana, basado en la media aritmética no ponderada y el algoritmo SAHN del paquete de programa NTSys versión 2.1.

\section{Resultados y discusión}

\subsection{Obtención de aislamientos}

De acuerdo a su origen y la variedad de mango con la que se trabajó se obtuvieron 22 aislamientos fúngicos que aparecen relacionados en la Tabla 1.

\subsection{Caracterización cultural de los aisla- mientos}

En general para las temperaturas de 20, 24, 27 y $30^{\circ} \mathrm{C}$ el crecimiento fue siempre circular y con bordes regulares excepto para los aislados 9 y 20 que presentaron bordes irregulares. Las coloraciones de las colonias variaron entre el blanco (Figura 1: D y E), gris verdoso (Figura 1: A y B) y gris pálido (Figura 1: C y F).

En la mayoría se encontraron sectores de crecimiento (Figura 1: A, C y D) y en muy pocos, halos concéntricos de crecimiento (Figura 1: B y E). Ambos tipos de zonación fueron también comúnmente observadas en el reverso de las colonias y en el caso de los aislamientos 4, 5, 11, 13 y 17 los sectores formaban una estrella. Se manifestaron diferentes tipos de texturas en las colonias: harinosa (Figura 1: F), lanosa (Figura1: A y B), algodonosa (Figura1: C y D) y afelpada (Figura1: E), pudiendo presentarse diferencias de textura del centro al margen de la colonia (Figura 1: A). Solo los aislados 10, 13, 14 y 15 presentaron pigmentos de color rosa salmón. La presencia de exudaciones sobre el micelio se manifestó de forma variable entre los aislados a las diferentes temperaturas por lo que no puede catalogarse como factor distintivo de una cepa o temperatura. Estos resultados coinciden con los reportados por 
Tabla 1. Relación de aislados de Colletotrichum sp. obtenidos de acuerdo a su localización y variedad de plantas de mangos

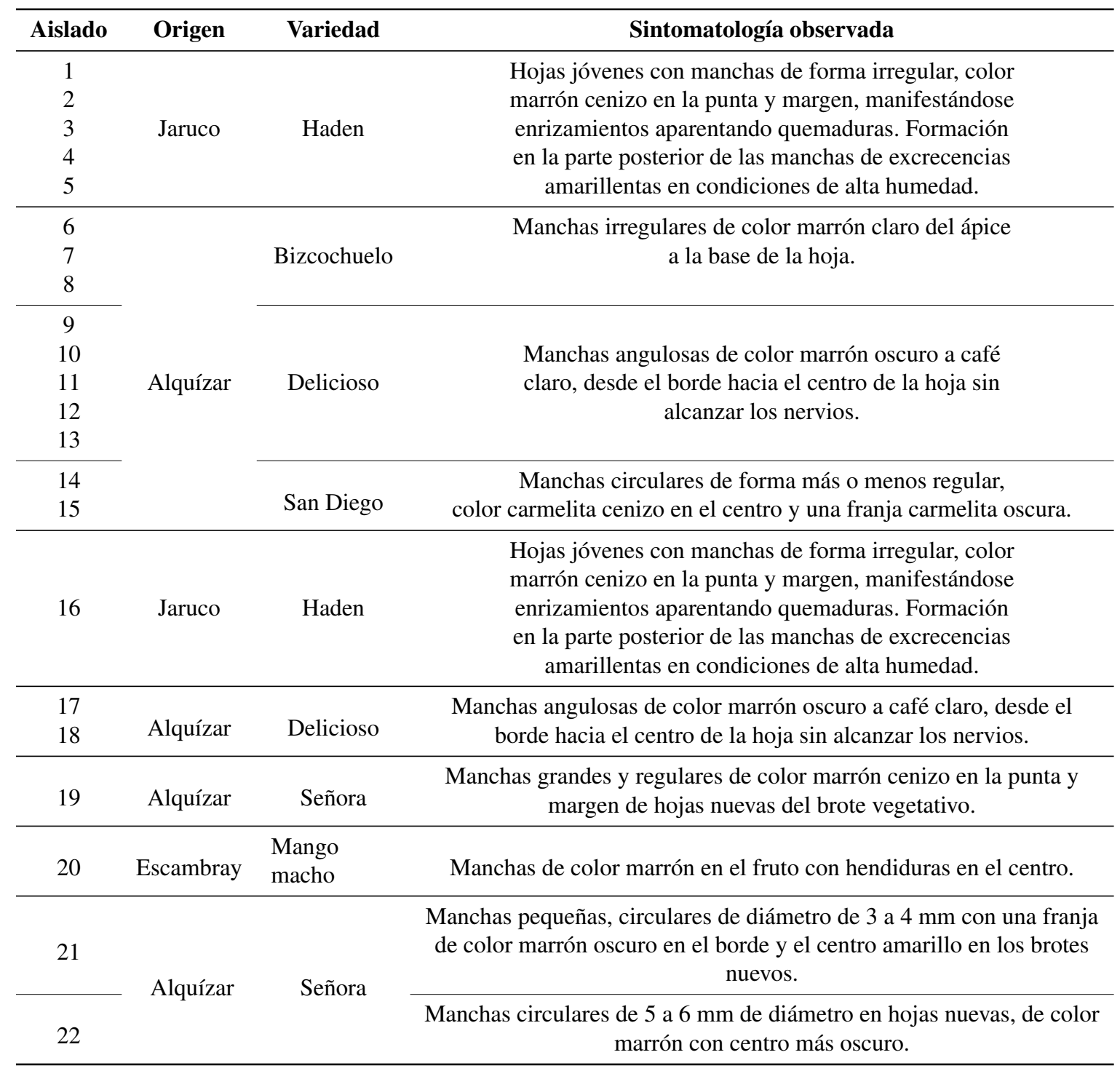



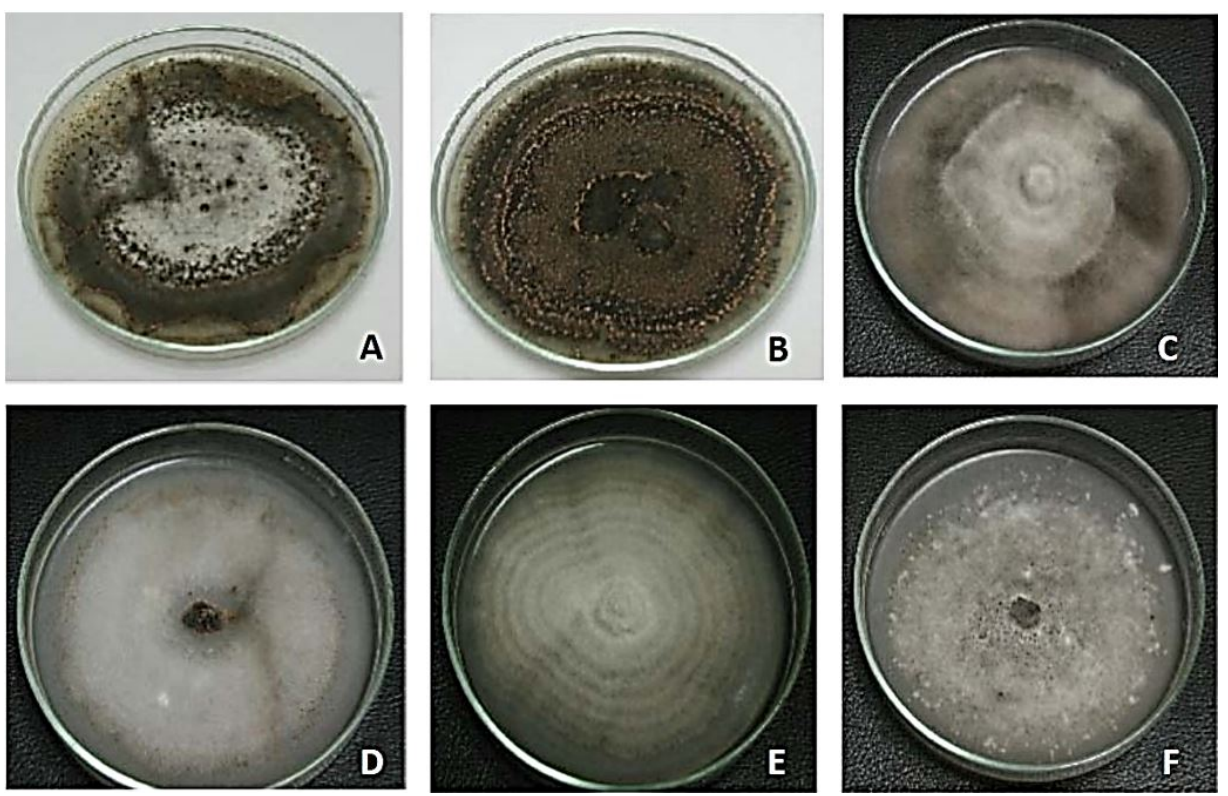

Figura 1. Diferentes tipos de crecimiento de las colonias de los aislados en caja Petri con PDA a $27 \pm 2^{\circ} \mathrm{C}$, después de 7 días incubación durante es estudio

Sangeetha y Rawal en el 2008 quienes obtuvieron aislamientos con semejantes características. La temperatura de $27^{\circ} \mathrm{C}$ favoreció la esporulación respecto a las restantes. La masa conidial se presentó principalmente como exudaciones de color naranja, viscosas que se desarrollaron a partir de acérvulos maduros sin setas; concordando con lo planteado por Arauz (2000) y Gutiérrez et al. (2001).

\subsection{Observación de la fase sexual}

En general, los aislamientos que formaron fase sexual se observaron pocos peritecios y la mayoría de ellos estaban inmaduros. Solo los aislamientos 2, 3 y 5 los presentaron de color negros y errumpentes en medio de cultivo.

La morfología y dimensión de las ascosporas permite establecer que las cepas periteciales pertenecen a la especie Glomrella cingulata (Stonem) de acuerdo a lo publicado por Freeman et al. (1998); Gutiérrez et al. (2001) y Carrillo et al. (2005). En los aislados 2 y 3 se observaron ascas maduras con ascosporas de 12-20 x 5-6 $\mu \mathrm{m}$, hialinas, redondeadas, multivacuoladas y curvadas (Cerón et al., 2007).

\subsection{Determinación de la concentración co- nidial}

Los resultados del conteo de conidios en Cámara de Neubauer aparecen en la Tabla 2. Se encontraron diferencias significativas $(\mathrm{P}<0.05)$ entre los aislamientos en el número de conidios producidos. En todos los casos la concentración de conidios se encontró por encima de $1 \times 105$ conidios. $\mathrm{ml}^{-1}$ coincidiendo con lo informado por Sanders et al., 2000 y Sangeetha y Rawal (2008). Sobresalen los aislados 4 y 5 con concentraciones del orden de 107 conidios. $\mathrm{ml}^{-1}$.

\subsection{Caracterización morfométrica de los aislamientos}

\subsubsection{Morfometría de los conidios}

Los aislamientos formaron conidios hialinos, cilíndricos y rectos. Su largo osciló entre los 15.7 y 11.1 $\mu \mathrm{m}$ y su ancho entre los 3.0 y $4.5 \mu \mathrm{m}$ (Tabla 3). En su mayoría presentaron ambos extremos redondeados aunque un porciento considerable presentó al menos un extremo ahusado lo que coincide con lo encontrado por Sanders et al. (2000) y Sangeetha y Rawal (2008). Todos los conidios presentaron abundantes cuerpos esféricos como publica Kuo (1999). En los aislados 6, 8, 13 y 19 entre el 1 y el 3\% de los conidios fueron fusiformes. 


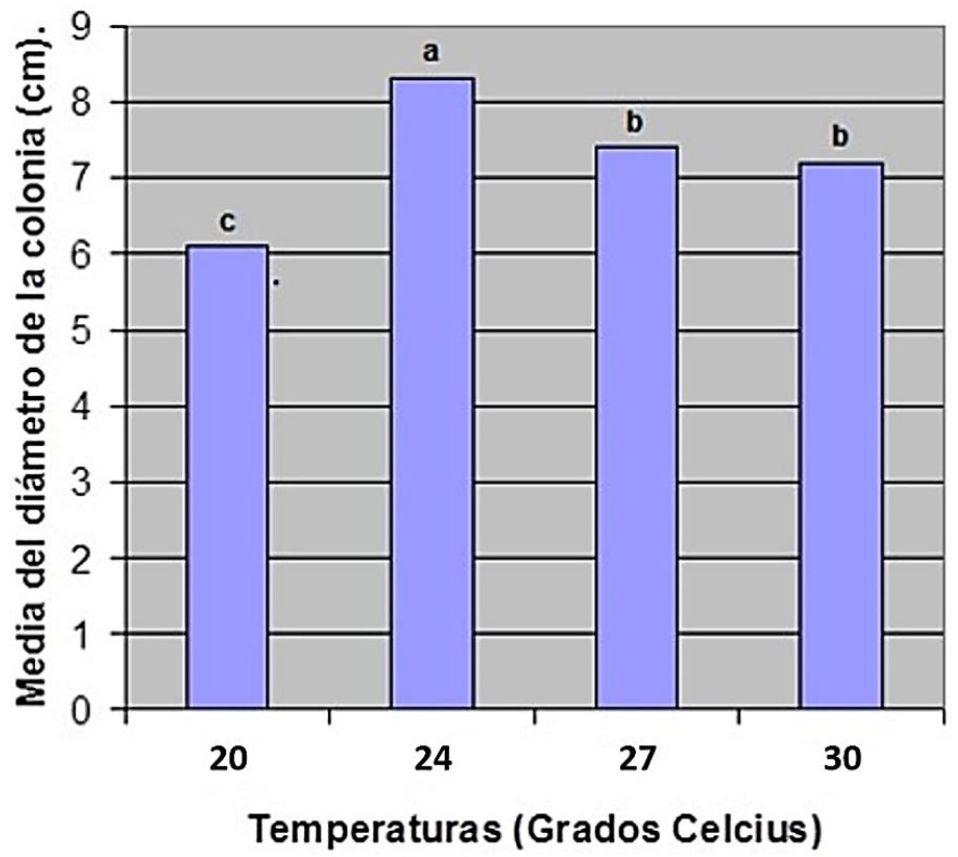

Figura 2. Media del crecimiento de microbiano medidos en centímetros en función a las diferentes temperaturas en medio de cultivo PDA (CV: 0.61. S̄̃ : 0.0443)

Tabla 2. Medida de la concentración de conidios por ml obtenido en Cámara de Neubauer a partir de colonias de 15 días de incubación a $30^{\circ} \mathrm{C} \pm 2{ }^{\circ} \mathrm{C}$ en medio de cultivo PDA con análisis estadístico de $\mathrm{P}<0.05$

\begin{tabular}{cl}
\hline Aislados & Cantidad de conidios por ml \\
\hline 5 & $1.47 \times 107 \mathrm{a}$ \\
4 & $1.14 \times 107 \mathrm{a}$ \\
17 & $7.75 \times 106 \mathrm{~b}$ \\
19 & $7.35 \times 106 \mathrm{~b}$ \\
11 & $5.00 \times 106 \mathrm{~b}$ \\
22 & $3.70 \times 106 \mathrm{~b}$ \\
21 & $3.60 \times 106 \mathrm{~b}$ \\
2 & $2.45 \times 106 \mathrm{~b}$ \\
1 & $1.75 \times 106 \mathrm{~b}$ \\
3 & $1.70 \times 106 \mathrm{~b}$ \\
7 & $1.35 \times 106 \mathrm{~b}$ \\
20 & $1.30 \times 106 \mathrm{~b}$ \\
9 & $9.00 \times 105 \mathrm{c}$ \\
16 & $9.00 \times 105 \mathrm{c}$ \\
8 & $7.50 \times 105 \mathrm{c}$ \\
13 & $7.00 \times 105 \mathrm{c}$ \\
6 & $6.00 \times 105 \mathrm{c}$ \\
15 & $5.00 \times 105 \mathrm{c}$ \\
18 & $5.00 \times 105 \mathrm{c}$ \\
10 & $4.00 \times 105 \mathrm{c}$ \\
14 & $3.00 \times 105 \mathrm{c}$ \\
12 & $2.00 \times 105 \mathrm{c}$ \\
\hline
\end{tabular}


Tabla 3. Relación de las medidas de las estructuras conidios y apresorios de los aislados con sus correspondientes criterios de forma expresados en porciento

\begin{tabular}{|c|c|c|c|c|}
\hline Aislado & $\begin{array}{l}\text { Conidios } \\
\text { Tamaño }(\mu \mathbf{m})\end{array}$ & $\begin{array}{l}\text { Extremos(fusiformes o re- } \\
\text { dondeados) }\end{array}$ & $\begin{array}{l}\text { Apresorios } \\
\text { Forma }\end{array}$ & Tamaño $(\mu \mathbf{m})$ \\
\hline 1 & $\begin{array}{l}11.2-14.4 \times 3.2-6.4 \\
x=12.1 \times 3.9\end{array}$ & $\begin{array}{l}89 \% \text { redondeados } \\
11 \% \text { ambos }\end{array}$ & $\begin{array}{l}60 \% \text { irregulares } \\
38 \% \text { clavados } \\
2 \% \text { redondeados }\end{array}$ & $\begin{array}{l}7.5-17.5 \times 5.0-10 \\
x=11.5 \times 6.7\end{array}$ \\
\hline 2 & $\begin{array}{l}9.6-14.4 \times 3.2-6.4 \\
x=12.0 \times 3.9\end{array}$ & $\begin{array}{l}88 \% \text { redondeados } \\
12 \% \text { ambos }\end{array}$ & $\begin{array}{l}80 \% \text { irregulares } \\
16 \% \text { clavados } \\
4 \% \text { redondeados }\end{array}$ & $\begin{array}{l}7.5-17.5 \times 5.0-10 \\
x=12.6 \times 6.7\end{array}$ \\
\hline 3 & $\begin{array}{l}11.2-14.4 \times 3.2-4.8 \\
x=11.9 \times 3.8\end{array}$ & $\begin{array}{l}92 \% \text { redondeados } \\
8 \% \text { ambos }\end{array}$ & $\begin{array}{l}89 \% \text { irregulares } \\
10 \% \text { clavados } \\
1 \% \text { redondeados }\end{array}$ & $\begin{array}{l}7.5-17.5 \times 5.0-12.5 \\
x=11.6 \times 6.8\end{array}$ \\
\hline 4 & $\begin{array}{l}11.2-14.4 \times 3.2-4.8 \\
x=12.4 \times 4.1\end{array}$ & $\begin{array}{l}85 \% \text { redondeados } \\
15 \% \text { ambos }\end{array}$ & $\begin{array}{l}94 \% \text { irregulares } \\
6 \% \text { clavados }\end{array}$ & $\begin{array}{l}7.5-17.5 \times 5.0-7.5 \\
x=11.7 \times 5.9\end{array}$ \\
\hline 5 & $\begin{array}{l}11.2-14.4 \times 3.2-4.8 \\
x=12.2 \times 4.5\end{array}$ & $\begin{array}{l}82 \% \text { redondeados } \\
18 \% \text { ambos }\end{array}$ & $\begin{array}{l}95 \% \text { irregulares } \\
3 \% \text { clavados } \\
2 \% \text { redondeados }\end{array}$ & $\begin{array}{l}7.5-12.5 \times 5.0-10 \\
x=10.5 \times 5.4\end{array}$ \\
\hline 6 & $\begin{array}{l}11.2-16 \times 1.6-4.8 \\
x=13.6 \times 3.3\end{array}$ & $\begin{array}{l}87 \% \text { ambos } \\
3 \% \text { fusiformes } \\
10 \% \text { redondeados }\end{array}$ & $\begin{array}{l}70 \% \text { irregulares } \\
29 \% \text { clavados } \\
1 \% \text { redondeados }\end{array}$ & $\begin{array}{l}8.6-21.5 \times 4.3-17.2 \\
\times=14.0 \times 9.9\end{array}$ \\
\hline 7 & $\begin{array}{l}9.6-14.4 \times 3.2-4.8 \\
x=11.1 \times 3.4\end{array}$ & $\begin{array}{l}90 \% \text { ambos } \\
10 \% \text { redondeados }\end{array}$ & $\begin{array}{l}80 \% \text { irregulares } \\
20 \% \text { clavados }\end{array}$ & $\begin{array}{l}8.6-21.5 \times 4.3-17.2 \\
x=14.3 \times 8.8\end{array}$ \\
\hline 8 & $\begin{array}{l}11.2-16 \times 1.6-6.4 \\
x=13.0 \times 3.5\end{array}$ & $\begin{array}{l}89 \% \text { ambos } \\
10 \% \text { redondeados } \\
1 \% \text { fusiformes }\end{array}$ & $\begin{array}{l}80 \% \text { irregulares } \\
18 \% \text { clavados } \\
2 \% \text { redondeados }\end{array}$ & $\begin{array}{l}12.5-21.5 \times 4.3-17.2 \\
x=14.5 \times 10.1\end{array}$ \\
\hline 9 & $\begin{array}{l}11.2-16.0 \times 3.2-4.8 \\
x=13.6 \times 3.8\end{array}$ & $\begin{array}{l}70 \% \text { redondeados } \\
30 \% \text { ambos }\end{array}$ & $\begin{array}{l}77 \% \text { irregulares } \\
15 \% \text { clavados } \\
8 \% \text { redondeados }\end{array}$ & $\begin{array}{l}7.5-17.5 \times 5.0-10 \\
x=11.5 \times 7.3\end{array}$ \\
\hline 10 & $\begin{array}{l}8.0-16.0 \times 3.2-4.8 \\
x=13.2 \times 4.2\end{array}$ & $\begin{array}{l}75 \% \text { redondeados } \\
25 \% \text { ambos }\end{array}$ & $\begin{array}{l}86 \% \text { irregulares } \\
14 \% \text { clavados }\end{array}$ & $\begin{array}{l}8.6-21.5 \times 4.3-17.2 \\
x=14.1 \times 9.5\end{array}$ \\
\hline 11 & $\begin{array}{l}11.2-16.0 \times 3.2-4.8 \\
x=13.9 \times 3.9\end{array}$ & $\begin{array}{l}79 \% \text { redondeados } \\
21 \% \text { ambos }\end{array}$ & $\begin{array}{l}76 \% \text { irregulares } \\
24 \% \text { clavados }\end{array}$ & $\begin{array}{l}7.5-21.5 \times 4.3-21.5 \\
x=12.2 \times 7.9\end{array}$ \\
\hline 12 & $\begin{array}{l}11.2-16.0 \times 3.2-4.8 \\
x=13.4 \times 4.1\end{array}$ & $\begin{array}{l}77 \% \text { redondeados } \\
23 \% \text { ambos }\end{array}$ & $\begin{array}{l}78 \% \text { irregulares } \\
20 \% \text { clavados } \\
2 \% \text { redondeados }\end{array}$ & $\begin{array}{l}8.6-17.2 \times 4.3-12.9 \\
x=13.8 \times 8.4\end{array}$ \\
\hline 13 & $\begin{array}{l}11.2-16.0 \times 3.2-4.8 \\
x=13.1 \times 3.9\end{array}$ & $\begin{array}{l}75 \% \text { redondeados } \\
24 \% \text { ambos } \\
1 \% \text { fusiformes }\end{array}$ & $\begin{array}{l}70 \% \text { irregulares } \\
28 \% \text { clavados } \\
2 \% \text { redondeados }\end{array}$ & $\begin{array}{l}8.6-21.5 \times 4.3-17.2 \\
x=13.9 \times 8.9\end{array}$ \\
\hline 14 & $\begin{array}{l}9.6-14.4 \times 3.0-3.2 \\
x=11.9 \times 3.2\end{array}$ & $\begin{array}{l}80 \% \text { ambos } \\
20 \% \text { redondeados }\end{array}$ & $\begin{array}{l}81 \% \text { irregulares } \\
19 \% \text { clavados }\end{array}$ & $\begin{array}{l}5.0-12.5 \times 2.5-12.5 \\
x=9.8 \times 6.6\end{array}$ \\
\hline 15 & $\begin{array}{l}8.0-16.0 \times 1.6-3.2 \\
x=11.3 \times 3.1\end{array}$ & $\begin{array}{l}88 \% \text { ambos } \\
12 \% \text { redondeados }\end{array}$ & $\begin{array}{l}83 \% \text { irregulares } \\
12 \% \text { clavados } \\
5 \% \text { redondeados }\end{array}$ & $\begin{array}{l}7.5-20.0 \times 5.0-10.0 \\
x=10.4 \times 7.2\end{array}$ \\
\hline 16 & $\begin{array}{l}8.0-16.0 \times 3.2-4.8 \\
x=11.2 \times 3.5\end{array}$ & $\begin{array}{l}72 \% \text { ambos } \\
28 \% \text { redondeados }\end{array}$ & $\begin{array}{l}89 \% \text { irregulares } \\
11 \% \text { clavados }\end{array}$ & $\begin{array}{l}8.6-21.5 \times 8.6-12.9 \\
x=13.8 \times 7.9\end{array}$ \\
\hline 17 & $\begin{array}{l}9.6-12.8 \times 1.6-4.8 \\
x=11.5 \times 3.3\end{array}$ & $\begin{array}{l}94 \% \text { redondeados } \\
6 \% \text { ambos }\end{array}$ & $\begin{array}{l}75 \% \text { irregulares } \\
25 \% \text { clavados }\end{array}$ & $\begin{array}{l}10.0-15.0 \times 5.0-10.0 \\
x=12.6 \times 7.4\end{array}$ \\
\hline 18 & $\begin{array}{l}8.0-16.0 \times 3.2-4.8 \\
x=12.7 \times 3.5\end{array}$ & $\begin{array}{l}95 \% \text { redondeados } \\
5 \% \text { ambos } \\
88 \% \text { ambos }\end{array}$ & $\begin{array}{l}70 \% \text { irregulares } \\
30 \% \text { clavados } \\
80 \% \text { irregular }\end{array}$ & $\begin{array}{l}7.5-12.5 \times 5.0-10.0 \\
x=10.6 \times 6.6\end{array}$ \\
\hline 19 & $\begin{array}{l}12.8-17.6 \times 3.2-6.4 \\
x=15.6 \times 3.5\end{array}$ & $\begin{array}{l}10 \% \text { redondeados } \\
2 \% \text { fusiformes }\end{array}$ & $\begin{array}{l}19 \% \text { clavados } \\
1 \% \text { redondeados }\end{array}$ & $\begin{array}{l}9.6-15.0 \times 5.0-11.2 \\
\times=12.4 \times 7.8\end{array}$ \\
\hline 20 & $\begin{array}{l}12.8-19.2 \times 1.6-3.2 \\
x=15.2 \times 3.0\end{array}$ & $\begin{array}{l}68 \% \text { ambos } \\
32 \% \text { redondeados }\end{array}$ & $\begin{array}{l}84 \% \text { irregular } \\
16 \% \text { clavados }\end{array}$ & $\begin{array}{l}7.5-12.8 \times 4.8-7.5 \\
x=9.7 \times 5.5\end{array}$ \\
\hline 21 & $\begin{array}{l}14.4-17.6 \times 3.2-4.8 \\
x=15.7 \times 3.3\end{array}$ & $\begin{array}{l}74 \% \text { ambos } \\
26 \% \text { redondeados }\end{array}$ & $\begin{array}{l}75 \% \text { irregular } \\
25 \% \text { clavados }\end{array}$ & $\begin{array}{l}6.4-12.8 \times 3.2-8.0 \\
x=10.8 \times 6.0\end{array}$ \\
\hline 22 & $\begin{array}{l}14.4-16.0 \times 3.2-4.8 \\
x=15.5 \times 3.6\end{array}$ & $\begin{array}{l}76 \% \text { ambos } \\
24 \% \text { redondeados }\end{array}$ & $\begin{array}{l}77 \% \text { irregulares } \\
23 \% \text { clavados }\end{array}$ & $\begin{array}{l}9.6-16.0 \times 4.8-8.0 \\
x=12.9 \times 6.3\end{array}$ \\
\hline
\end{tabular}

LA GRANJA:Revista de Ciencias de la Vida 26(2) 2017:38-51. 


\subsection{Morfometría de los apresorios}

Se observaron apresorios lobulados o irregulares, clavados y ovoides, de color pardo oscuro. La forma irregular predominó sobre las restantes como aparece reflejado en la Tabla 3. En todos los casos se observaron apresorios terminales en tubos germinativos con tallas en un rango de 9.7-14.5 x 5.5-10.1 $\mu \mathrm{m}$. Esto coincide con Páez (1996); Arauz (2000) y Sangeetha y Rawal (2008) quienes observaron apresorios lobulados, pardo oscuros y que podían ser terminales en tubos germinativos o salir directamente de los conidios.

Estas características coinciden con la información registrada en la descripción de la especie Colletotrichum gloeosporiodes (Penz) Penz \& Sacc por Sutton (1980), quien define que esta presenta conidios rectos, cilíndricos, de 9.0-24.1 x 3.0-6.7 $\mu \mathrm{m}$ y apresorios de 6-21 x 4-12.7 $\mu \mathrm{m}$, clavados o irregulares. Por otra parte, Carrillo et al. (2005) plantean que la mayoría de los aislados de Colletotrichum gloeosporioides forman conidios con ápices redondeados y menos del 10\% con ápices ahusados.

\subsection{Prueba de patogenicidad}

El estudio in vitro de la virulencia de los 22 aislados mostró que las infecciones experimentales fueron positivas, ejemplos ilustrados en la Figura 3. Las hojas mostraron manchas de color marrón, con forma regular, las mismas aumentaron gradualmente y en algunos casos llegaron a cubrir totalmente el órgano de la planta durante el tiempo de estudio. Al cabo de los 5 días, en las lesiones hubo crecimiento micelial emergente y aparecieron acérvulos con abundante producción de conidios inmersos en masas cromógenas, anaranjadas en su mayoría y salmón solo en el caso del aislado 21 . En ningún caso se produjeron peritecios propios de la especie Glomrella cingulata (Stonem) Spauld \& Schrenk. Los materiales usados como testigos permanecieron siempre libres de infección.

Las lesiones con mayor velocidad de crecimiento para todas las variedades fueron las provocadas por las cepas $21,19,3,2,17$ y 5 en ese orden respectivamente. Dentro de estas se encuentran tanto cepas periteciales como productoras únicamente de conidios, contrastando con los resultados obtenidos por Gutiérrez et al. (2001) quienes plantean que las lesiones causadas por la cepa peritecial, crecen más rápidamente que las ocasionadas por la conidial.
Se observaron diferencias significativas $(\mathrm{P}<0.05)$ entre las variedades (Figura 4). La variedad Filipino fue la más afectada contraponiéndose a lo planteado por Cañizares (1966) y Capote et al. (1989) quienes la incluyen dentro de las más resistentes a la antracnosis. Estas diferencias respecto a las demás variedades puede deberse a que el la variedad de mango Filipino es considerada por los agricultores como una variedad temprana, que su período de maduración comienza en el mes de abril, quizás esta baja susceptibilidad está referida no a una resistencia genética sino a que los frutos se producen y maduran temprano, fuera de la época de lluvias en que se favorece la infección por el patógeno, por tanto, no contradicen los resultados obtenidos. La variedad Señora mostró una alta susceptibilidad al patógeno coincidiendo con Capote et al. (1989).

Entre las variedades Haden y Keitt no hubo diferencia significativa. Ambas presentaron "in vitro"bajo nivel de susceptibilidad. Estos resultados coinciden con lo planteado por Capote et al. (1989), para quien estas se agrupan dentro de las variedades con baja susceptibilidad a la antracnosis en el campo. Sin embargo, Campbell (1992) y Rodríguez et al. (2002) publican sensibilidad a la enfermedad para la variedad Haden. Estas divergencias pueden tener su base en la influencia de las diferencias climáticas sobre el ciclo de la enfermedad y la maduración del fruto, debido a que los estudios se realizaron en diferentes regiones.

La virulencia de los aislados es presentada en la Tabla 4, donde los menos patogénicos son aquellos que producen menor cantidad de conidios teniendo en cuenta los resultados del conteo realizado. Los aislados obtenidos de Haden y Señora fueron más patogénicos en estas que en el resto de las variedades, concordando con Sangeetha y Rawal (2008) y Denoyes et al. (2003) quienes plantean que las cepas obtenidas de un hospedero particular eran más patogénicas en este que en otros hospederos.

Se reprodujeron los mismos síntomas observados en las muestras del campo y los reaislamientos mostraron las mismas características que los originales. Según los postulados de Koch, las cepas aisladas fueron las causantes de la antracnosis en las muestras colectadas. 

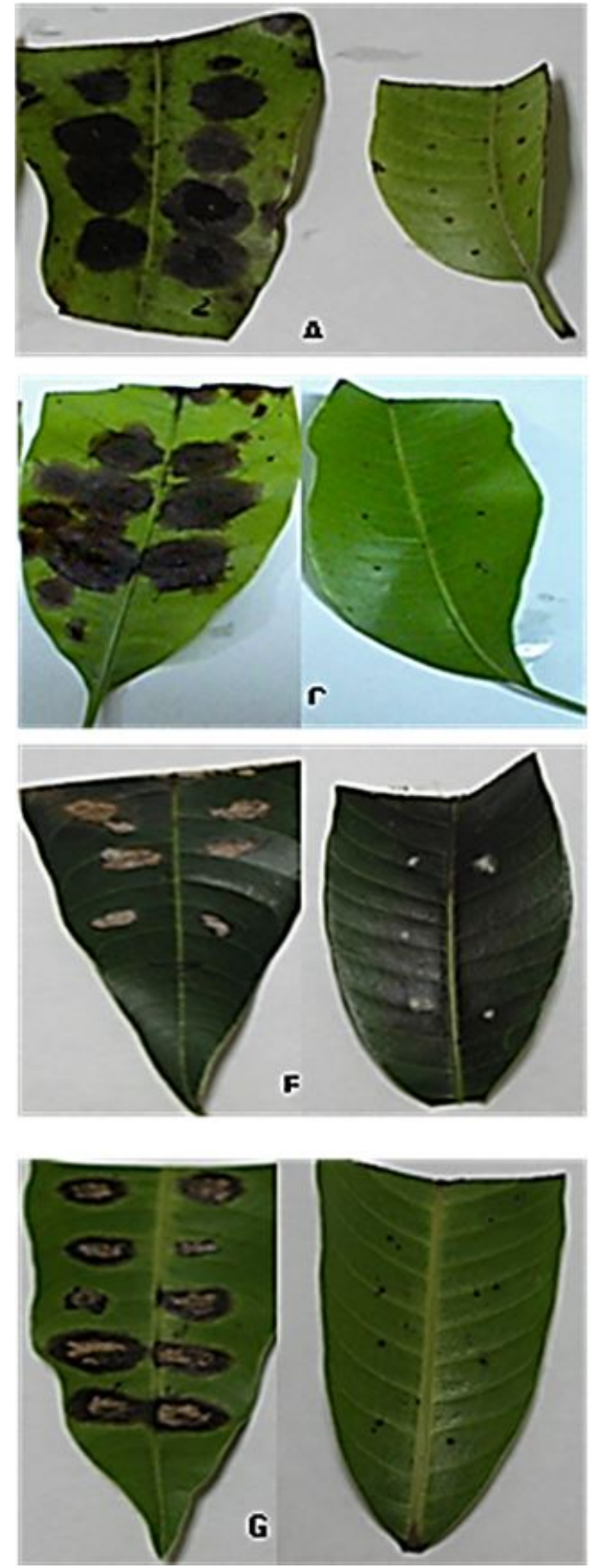
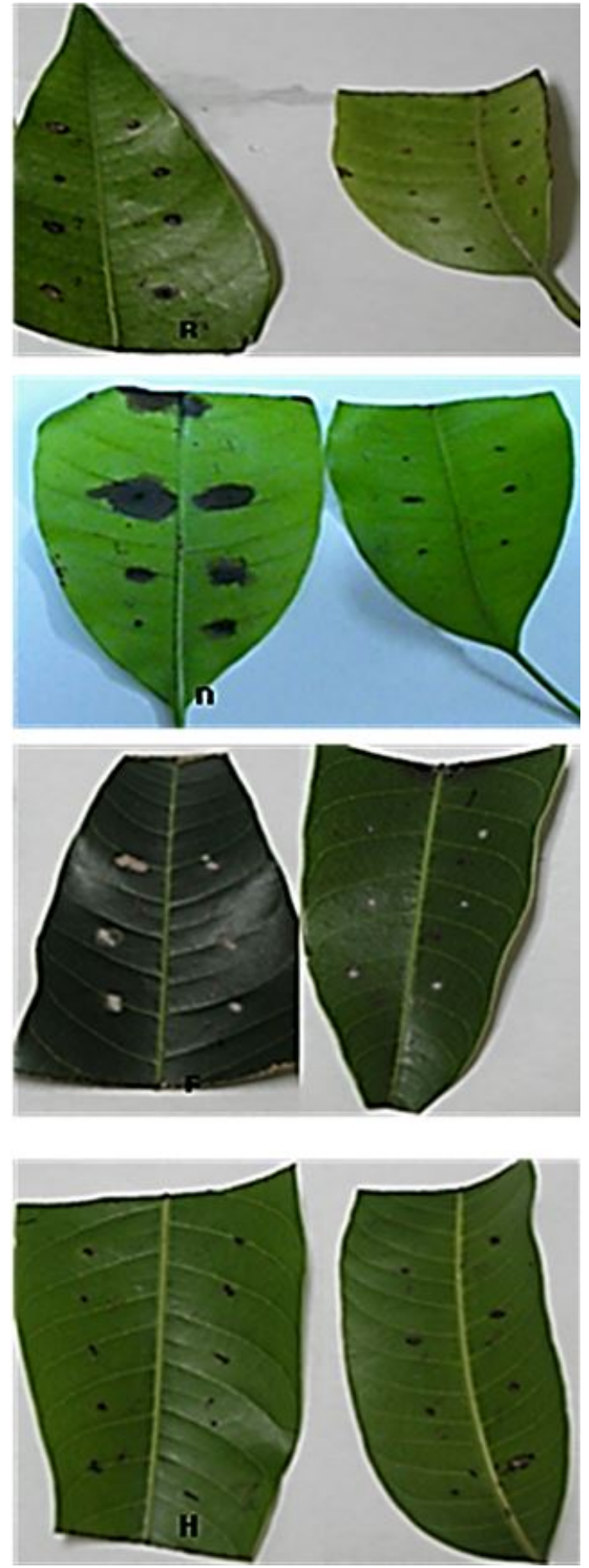

Figura 3. Pruebas in vitro de la patogenicidad de los 22 aislados y sus correspondientes testigos en hojas de diferentes variedades de mangos. (A-aislado 21 variedad Filipino; B-aislado 7 variedad Señora; C-aislado 13 variedad Filipino; D-aislado 10 variedad Keitt; E-aislado 12 variedad Haden; F-aislado 20 variedad Haden; G-aislado 3 variedad Señora y H-aislado 6 variedad Keitt). 


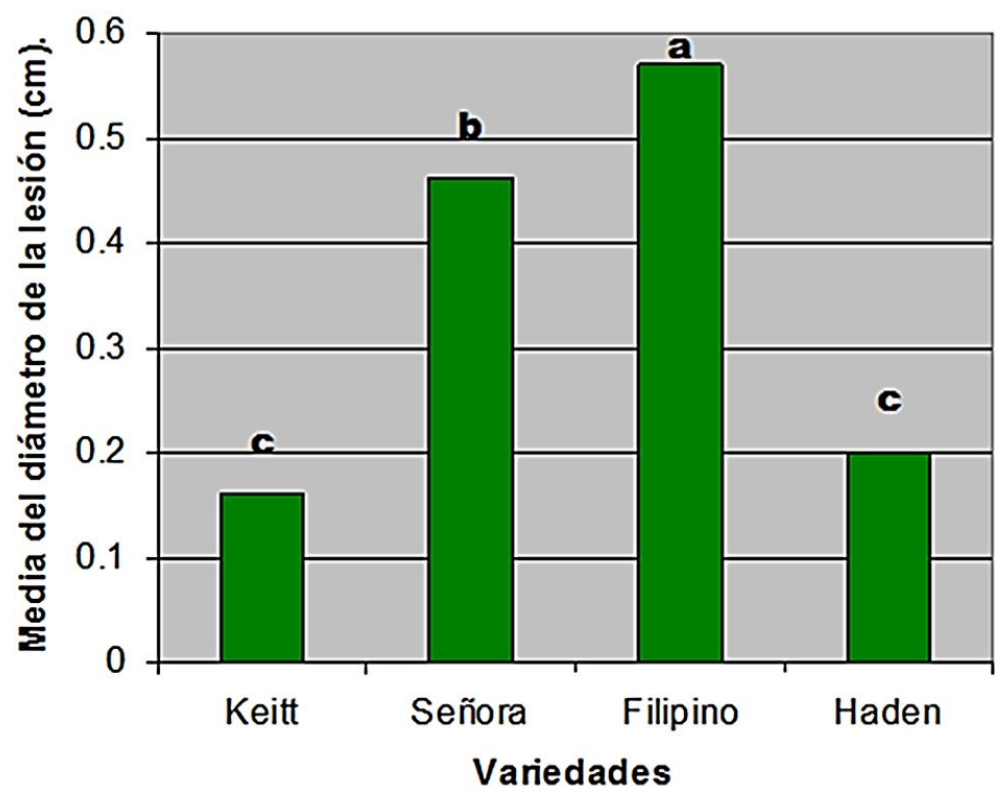

Figura 4. Medida de la sensibilidad de las variedades de mango resultado de las pruebas de patogenicidad de los 22 aislados de Colletotrichum gloeosporiodes (Penz) Penz \& Sacc.

Tabla 4. Relación de las medidas de las estructuras conidios y apresorios de los aislados con sus correspondientes criterios de forma expresados en porciento

\begin{tabular}{ccc}
\hline Aislado & Dato original & Dato transformado \\
\hline $\mathbf{2 1}$ & 0.69 & $1.69 \mathrm{a}$ \\
$\mathbf{1 7}$ & 0.59 & $1.59 \mathrm{~b}$ \\
$\mathbf{3}$ & 0.55 & $1.55 \mathrm{~b}$ \\
$\mathbf{2 2}$ & 0.54 & $1.54 \mathrm{~b}$ \\
$\mathbf{1}$ & 0.53 & $1.53 \mathrm{~b}$ \\
$\mathbf{1 9}$ & 0.51 & $1.51 \mathrm{c}$ \\
$\mathbf{2}$ & 0.50 & $1.50 \mathrm{bc}$ \\
$\mathbf{5}$ & 0.49 & $1.49 \mathrm{bc}$ \\
$\mathbf{1 8}$ & 0.41 & $1.41 \mathrm{~cd}$ \\
$\mathbf{1 0}$ & 0.37 & $1.37 \mathrm{~d}$ \\
$\mathbf{2 0}$ & 0.34 & $1.34 \mathrm{~d}$ \\
$\mathbf{4}$ & 0.33 & $1.33 \mathrm{~d}$ \\
$\mathbf{1 6}$ & 0.22 & $1.22 \mathrm{e}$ \\
$\mathbf{1 5}$ & 0.20 & $1.20 \mathrm{e}$ \\
$\mathbf{1 4}$ & 0.19 & $1.19 \mathrm{e}$ \\
$\mathbf{1 1}$ & 0.19 & $1.19 \mathrm{e}$ \\
$\mathbf{1 3}$ & 0.17 & $1.17 \mathrm{e}$ \\
$\mathbf{7}$ & 0.17 & $1.17 \mathrm{e}$ \\
$\mathbf{9}$ & 0.15 & $1.15 \mathrm{e}$ \\
$\mathbf{1 2}$ & 0.14 & $1.14 \mathrm{e}$ \\
$\mathbf{8}$ & 0.14 & $1.14 \mathrm{e}$ \\
$\mathbf{6}$ & 0.12 & $1.12 \mathrm{e}$ \\
\hline
\end{tabular}


Tabla 5. Relación de las medidas de las estructuras conidios y apresorios de los aislados con sus correspondientes criterios de forma expresados en porciento

\begin{tabular}{lllll}
\hline Variables & CP1 & CP2 & CP3 & CP4 \\
\hline Color de la colonia & 0.375 & 1.16 & $\mathbf{- 0 . 7 3 2}$ & -0.096 \\
Cantidad de conidios & 0.418 & $\mathbf{- 0 . 7 1 9}$ & -0.233 & 0.216 \\
Exudaciones & 0.0091 & $\mathbf{0 . 7 5 9}$ & -0.241 & 0.311 \\
Forma de apresorio & 0.146 & -0.266 & $\mathbf{- 0 . 7 0 4}$ & -0.261 \\
Color de la masa conidial & $\mathbf{- 0 . 7 7 4}$ & 0.160 & 0.042 & -0.401 \\
\hline
\end{tabular}

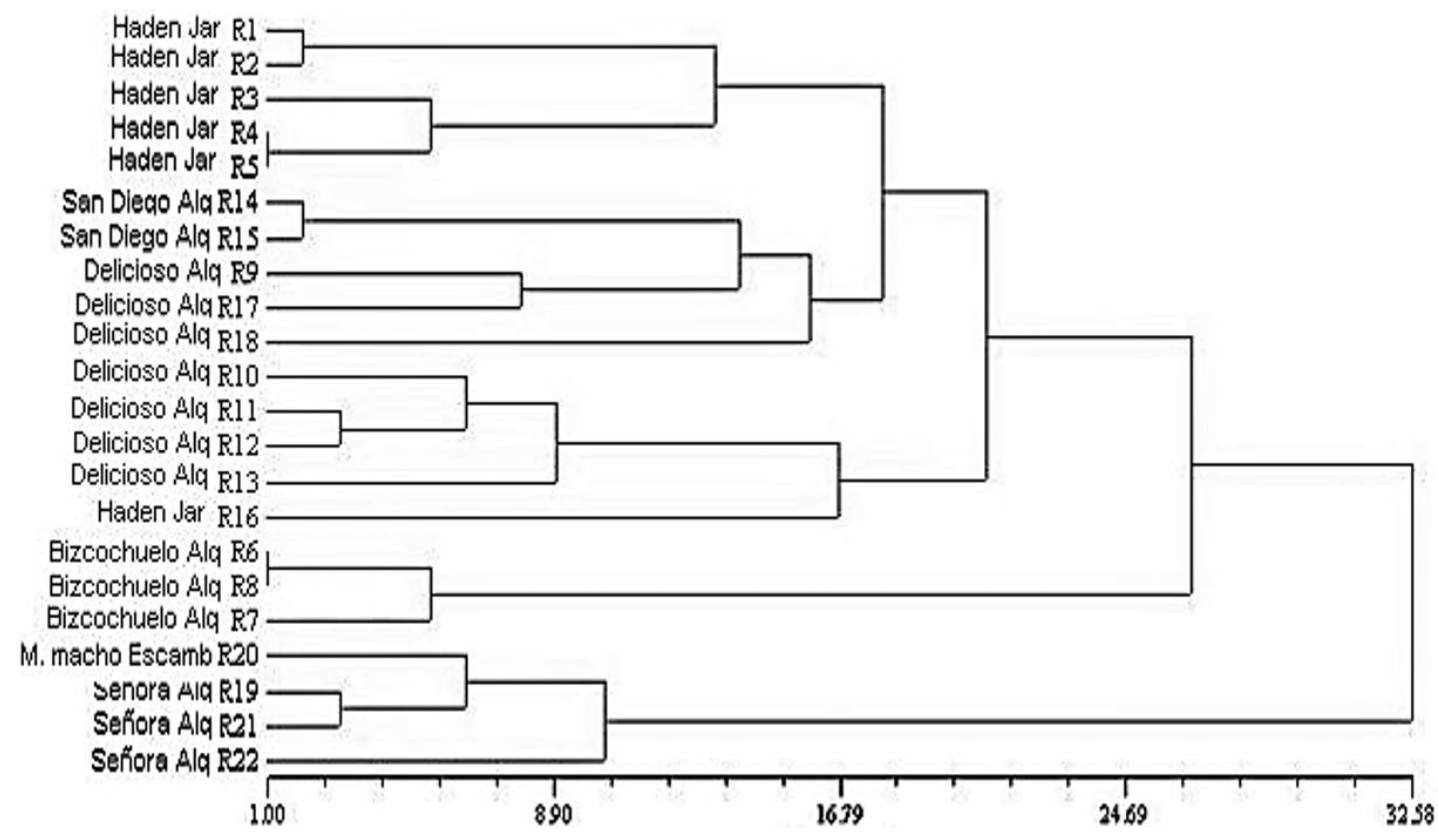

Figura 5. Agrupamiento de los 22 aislados de Colletotrichum gloeosporiodes (Penz) Penz \& Sacc. obtenidos de las seis variedades de mango en las diferentes localidades del occidente de Cuba. 


\subsection{Análisis estadístico para la diferencia- ción de los grupos}

Los factores que más influyen en el agrupamiento de los aislados se corresponden con el color de la colonia, la cantidad de conidios, las exudaciones al medio, la forma del apresorio y el color de la masa conidial como se muestra en la Tabla

La Figura 5 nos muestra el dendograma de los aislados de C. goleosporioides; estos resultados indican que los aislados se agrupan fundamentalmente atendiendo a la localidad de donde fueron obtenidos en la investigación. En el primer grupo (I) formado por los aislados de Jaruco var Haden que se diferencian perfectamente del grupo (II), quienes en su mayoría están constituidos por los aislados obtenidos a partir de diferentes variedades de mango colectadas en la localidad de Alquilar. Existe un aislamiento procedente del Escambray que se agrupa al final y no se corresponde con ninguno de los grupos mencionados anteriormente. En este sentido coincidimos con Torres (1995), quien planteó que las diferentes regiones climáticas de Cuba determinaban el comportamiento del cultivo, Gutiérrez et al. (2001), en estudios realizados afirmó que existen aislados de C.gloeosporioides que se agrupan más que por su variabilidad por la zona donde se realizó el aislamiento, indicando que el ambiente puede modificar el comportamiento de las cepas.

\section{Conclusiones}

Las 22 cepas aisladas en la región occidental de $\mathrm{Cu}$ ba, pertenecen a la especie Colletotrichum gloeosporioides (Penz.) Penz. and Sacc con una temperatura óptima de crecimiento por encima de $24 \pm$ 2oC. Las dimensiones de las estructuras medidas: conidios y apresorios, se encuentran entre los límites establecidos mundialmente para esta especie. La patogenicidad in vitro de los aislados se manifiesta en concordancia de la variedad de planta, siendo el aislado 21 con más grado de virulencia.

\section{Referencias}

Abd-Alla, M., y Wafaa, M. 2010. New Safe Methods for Controlling Anthracnose Disease of Mango (Mangifera indica L.) Fruits Caused by Colletotrichum gloeosporioides (Penz.). Journal of
American Science 8(8). p. 361-367. Disponible en: https://goo.gl/RhVDFE.

álvarez, E., Mejía, J., Llano, G. y Loke, J. 2006. Characterization of Colletotrichum gloeosporioides, causal agent of anthracnose in soursop (Annona muricata) in Valle del Cauca. Poster: International Center for Tropical Agriculture (CIAT). Disponible en: https://goo. $\mathrm{gl} / \mathrm{sCPevF}$

Arauz, L.F. 2000. Mango anthracnose: Economic impact and current options for integrated management. Plant Disease, 84(6), p. 600-611. DOI: https://doi.org/10.1094/PDIS.2000.84.6.600.

Bruwer, T., Le Legadec, M. D. y Köhne, J. S. 2006. A Decade of Mango Cultivar Evaluation by Westfalia Technological Services. 8th International Mango Symposium. South Africa Feb. 510: 113-118.

Butt, T., y Copping, L. 2000. Fungal biological control agents. Pesticide Outlook, 11, 186-191. DOI: https://doi.org/10.1039/B008009H

Campbell, R. J. 1992. A Guide to Mangos in Florida. Fairchild Tropical Garden, Miami, Florida. E.E. U.U.

Caǹizares, J. 1966. El Mango. INRA, 6-14, 42-44. La Habana, Cuba. Disponible en: https:/ /goo. $\mathrm{gl} / \mathrm{LvaRs} 7$

Capote, M., Lima, H., Cabrera, A.M., Naviera, A. y Blanco, M. 1989. Agrupación fenotípica de 31 cultivares de mango sobre la base de variables morfológicas y de resistencia a enfermedades. 'emphCienc. Tec. Agric. Cítricos y otros frutales, 12(4), p. 12-18. Disponible en: https://goo.gl/F5XJWn

Carrillo, J. A., García, R., Muy, M. D., Saǹudo, A., Márquez, I., Allende, R., De La Garza, Z., Patiǹo, M., y Galindo, E. 2005. Control biológico de la antracnosis (Colletotrichum gloeosporioides (Penz) Penz \& Sacc) y su efecto en la calidad poscosecha del mango (Mangífera índica L) en Sinaloa, México. Rev. Mexicana de Fitopatología, 23(1), p. 24-32. Disponible en: https://goo.gl/pQUKAZ

Cerón, L., Higuera, B., Sánchez, J., Bustamante, S., y Buitrago, G. 2007. Crecimiento y desarrollo de Colletotrichum gloeosporioides $\mathrm{f}$. alatae 
durante su cultivo en medios líquidos. Acta Biológica Colombiana, 11(1), p. 99-109. Disponible en: http://www.redalyc.org/articulo.oa? id $=319028578008$

Denoyes, B., Guérin, G., y Délye, C. 2003. Genetic diversity and pathogenic variability among isolates of Colletotrichum species from strawberry. Phytopathology, 93(2), p. 219-28. DOI: https://doi.org/10.1094/PHYTO.2003. 93.2.219.

Freeman, S., Katant, T., y Shabi, E. 1998. Characterization of Collectotrichum species responsible for anthracnose disease of various fruits. Plant Disease, 82(6), p. 596-605. DOI: https:// doi.org/10.1094/PDIS.1998.82.6.596

Gutiérrez, J., Nieto, D., Téliz, D., Zavaleta, E., Vaquera, H., Martínez, T., y Delgadillo, F. 2001. Características del crecimiento, esporulación y patogenicidad de aislamientos de Colletotrichum gloeosporioides (Penz) obtenidos de frutos de mango (Mangífera índica L). Rev. Mexicana de Fitopatología, 19(1), p. 90-93. Disponible en: https:/ /goo.gl/af86Tz

INFOAGRO 2006. El mercado de las frutas tropicales en la Unión Europea. 10pp. Disponible en: https://goo.gl/L1xuoY

Jayasinghe, C., y Fernando, T. 2009. First Report of Colletotrichum acutatum on Mangifera indica L in Sri Lanka. Ceylon Journal of Science (Biological Sciences), 38(1). p.31-34DOI: http: //doi.org/10.4038/cjsbs.v38i1.1326

Kuo, K. C. 1999. Germination and appressorium formation in Colletotrichum gloeosporioides. Proc. Natl. Sci. Counc. ROC (B), 23(3), p. 126132. Disponible en: https://goo.gl/foWSqe

Páez, A.R. 1996. Respuesta de variedades de mango (Mangífera índica L) a la antracnosis (Colletotrichum gloeosporioides). Fitopatología Colombiana, 20(1), p. 11-19. Disponible en: https://goo.gl/2BzmmK

Rebouca, A. 2002. Aspectos sobre la producción de mango. Curso Internacional de mango. Ciudad de Guatemala. Guatemala: 20-23.
Rodríguez, M., Guerrero, M., y Sandoval, R. 2002. Cultivo del mango. Guía Técnica. Centro Nacional de Tecnología Agropecuaria y Forestal (12).

Sanders, G., Korsten, L. y Wehner, F. 2000. Market survey of post harvest diseases and incidence of Colletotrichum gloeosporioides on avocado and mango fruit in South Africa. Trop. Sci., 40(4), p. 192-198. Disponible en: https: //goo.gl/g6oVuU

Sangeetha, C. G, y Rawal, R. D. 2008. Nutritional Studies of Colletotrichum gloeosporioides (Penz.) Penz. and Sacc. The Incitant of Mango Anthracnose. World Journal of Agricultural Sciences, 4(6), p. 717-720. Disponible en: https: //goo.gl/jqHXmj

Sigarroa, A. 1985. Biometría y diseǹo experimental. La Habana: Editorial Pueblo y Educación

Silva, K., Hojo, T., Lacerda, O., Pereira, M., Alves, A., Luna, G., Prado, A., RebouÃßas São, A., Oliveira, N., y Macedo, G. 2005. Patogenicidade causada pelo fungo Colletotrichum gloeosporioides (Penz) em diferentes espécies frutíferas. Rev. Bras. Frutic., Jaboticabal, 28(1), p. 131-133. Sao Pablo. Disponible en: https: //goo.gl/NXpvqs

Sutton, B. C. 1980. The Coelomycetes. Fungi Imperfecti with Pycnidia Acervuli and Stromata. Commonwealth Mycological Institute. Assoc. Appl. Biol., Kew, Surrey, England. Disponible en: https://goo.gl/qGpbUV

Torres, M. 1995. Resultados obtenidos en las investigaciones. Estación Nacional de Frutales. MINAGRI: 18-29.

Valdés, L., Gómez, A., Carballo, M., Capote, M., González. I. y Rohde, W. 2015. Estandarización de protocolos para la extracción de ADN cromosómico en cepas de Colletotrichum gloeosporioides aislados en plantas de mango (Mangifera indica L.). La Granja: Revista de Ciencias de la Vida, 22(2), p. 40-49. DOI: http:/ /dx.doi.org/10.17163/lgr.n22.2015.04 